\title{
Multicomponent Therapeutics of Berberine Alkaloids
}

\author{
Jiaoyang Luo,, ${ }^{1,2}$ Dan Yan, ${ }^{1,3}$ Meihua Yang, ${ }^{2}$ Xiaoping Dong, ${ }^{3}$ and Xiaohe Xiao ${ }^{1}$ \\ ${ }^{1}$ China Military Institute of Chinese Medicine, Integrative Medical Center of 302 Military Hospital, Beijing 100039, China \\ ${ }^{2}$ Institute of Medicinal Plant Development, Chinese Academy of Medical Sciences, Peking Union Medical College, Beijing 100193, China \\ ${ }^{3}$ College of Pharmacy, Chengdu University of Chinese Traditional Medicine, Chengdu 610075, China
}

Correspondence should be addressed to Xiaohe Xiao; pharmasci@126.com

Received 10 December 2012; Revised 16 February 2013; Accepted 24 February 2013

Academic Editor: Yao Tong

Copyright (C) 2013 Jiaoyang Luo et al. This is an open access article distributed under the Creative Commons Attribution License, which permits unrestricted use, distribution, and reproduction in any medium, provided the original work is properly cited.

\begin{abstract}
Although berberine alkaloids (BAs) are reported to be with broad-spectrum antibacterial and antiviral activities, the interactions among BAs have not been elucidated. In the present study, methicillin-resistant Staphylococcus aureus (MRSA) was chosen as a model organism, and modified broth microdilution was applied for the determination of the fluorescence absorption values to calculate the anti-MRSA activity of BAs. We have initiated four steps to seek the optimal combination of BAs that are (1) determining the anti-MRSA activity of single BA, (2) investigating the two-component combination to clarify the interactions among BAs by checkerboard assay, (3) investigating the multicomponent combination to determine the optimal ratio by quadratic rotationorthogonal combination design, and (4) in vivo and in vitro validation of the optimal combination. The results showed that the interactions among BAs are related to their concentrations. The synergetic combinations included "berberine and epiberberine," "jatrorrhizine and palmatine" and "jatrorrhizine and coptisine"; the antagonistic combinations included "coptisine and epiberberine". The optimal combination was berberine : coptisine : jatrorrhizine : palmatine : epiberberine $=0.702: 0.863: 1: 0.491: 0.526$, and the potency of the optimal combination on cyclophosphamide-immunocompromised mouse model was better than the natural combinations of herbs containing BAs.
\end{abstract}

\section{Introduction}

To seek a multicomponent drug from traditional medicine is one of the research directions for treating MRSA infections. Rhizoma Coptidis has been used for more than two thousand years in China, and it was known as "the antibiotics in traditional Chinese medicine". Although many studies were conducted on antimicrobial activities of berberine alkaloids (BAs) in the past few decades [1-7], few of them paid attention to the interactions among BAs. As we know, BAs are the active components of several traditional medicines, such as Rhizoma coptidis, Phellodendri Chinensis, and Berberidis Radix $[8,9]$, and the awareness of the interactions among BAs is of great value for the exploitation of such multicomponent drugs.

In the present study, methicillin-resistant Staphylococcus aureus (MRSA) was chosen as a model organism to study the interactions among BAs. MRSA is one of the pathogenic clinically separated strains leading to high morbidity and mortality [10]. Due to its multidrug-resistant genotype,
MRSA is not sensitive to the $\beta$-lactam antibiotics, and it is also resistant to aminoglycosides, fluoroquinolones, chloramphenicol, and macrolides [11-13], but not glycopeptides such as vancomycin and teicoplanin [14]. Single-drug treatment modalities have slowed down into bottle neck, alternatively the multi-component natural medicine might be one of the directions of the future antibacterial drug development [15]. We are expecting to clarify the synergetic and antagonistic actions and to seek the optimal combination of BAs which manifests the strongest anti-MRSA activity. We are initiating four steps to seek the optimal combination of BAs, that is, (1) determining the anti-MRSA of single BA, (2) investigating the two-component combination to clarify the interactions among BAs by checkerboard assay, (3) investigating the multi-component combination to determine the optimal ratio by quadratic rotation-orthogonal combination design, and (4) validation of the optimal combination.

There are several methods for the compatibility study such as tubes serial dilution method and agar checkerboard dilution method. Among these methods, checkerboard 
microdilution is one of the commonly used assays for twocomponent combinations that can quickly screen the antimicrobial activity [16]. The detection sensitivity of this method could be much improved by adding the fluorescent dye of resazurin, which makes it an accurate method for determining the anti-MRSA activity of BAs. However, checkerboard microdilution is not suitable for the investigation of multicomponent combinations, and hence the quadratic rotationorthogonal combination design (QRCD) was adopted to seek optimal combinations. Compared to unifactor experiment and orthogonal design, QRCD basically retained the advantages of less experiments, simple calculation and absence of the correlation between regression coefficients. In addition, the optimal region of the concentration could be directly obtained $[17,18]$. This study can help to clarify the interactions among BAs and provide a new idea for the exploitation of multi-component drugs.

\section{Materials and Methods}

2.1. Materials. Rhizoma Coptidis, Phellodendri Chinensis, and Berberidis Radix were collected in the place of production. Berberine (Ber, LOT: 110713-200911) and palmatine (Pal, LOT: 110732-200907), with the purity of $\geq 98 \%$, were provided by National Institutes for Food and Drug Control. Jatrorrhizine (Jat), coptisine (Cop), and epiberberine (Epi), with the purity of $\geq 97 \%$, were purchased from Zelang Medical Technology Co., Ltd., Nanjing, China. Their structures were given in Figure 1. All other chemicals used were of analytical grade and available locally.

2.2. Culture. MRSA Srain (ATCC43300), Escherichia coli (E. coli, CCTCC AB91112), Staphylococcus aureus (S. aureus, CCTCC AB910393), Shigella dysenteriae (S. dysenteriae, CCTCC AB210562), Streptococcus pneumoniae (S. pneumonia, ATCC49619), and Candida albicans (C. albicans, CCTCC C091101) were provided by China Center for Type Culture Collection and were cultivated in microbiological laboratory of 302 Military Hospital of China, Beijing, China. Briefly the broth culture medium contained $10 \mathrm{~g}$ peptone, $6 \mathrm{~g}$ beef extract, and $5 \mathrm{~g} \mathrm{NaCl}$ dissolving in $1000 \mathrm{~mL}$ deionized water ( $\mathrm{pH}$ 7.0-7.2). Then, this culture medium was sterilized in high-pressure steam $(0.1 \mathrm{MPa})$ at $121^{\circ} \mathrm{C}$ for $30 \mathrm{~min}$. Initially, the strains were inoculated into $25 \mathrm{~mL}$ broth culture medium in $100 \mathrm{~mL}$ wide-mouthed glass bottle and incubated in a shaker for $8 \mathrm{~h}$ at $37^{\circ} \mathrm{C}$. The rotation speed of incubator shaker was $110 \mathrm{rpm}$. The flask was enveloped with a cotton plug, so there was enough oxygen to be used by the strains. For the experiment, the bacteria were transformed and grown in cation-adjusted Mueller Hinton broth (CAMHB), which was prepared from $17.5 \mathrm{~g}$ casein acid hydrolysate, $3 \mathrm{~g}$ beef extract, and $1.5 \mathrm{~g}$ starch dissolving in $1000 \mathrm{~mL}$ distilled water (final $\mathrm{pH}$ 7.1-7.5 at $25^{\circ} \mathrm{C}$ ). Then, this culture medium was sterilized in high-pressure steam $(0.1 \mathrm{MPa})$ at $121^{\circ} \mathrm{C}$ for $30 \mathrm{~min}$ and stored in a refrigerator at $4^{\circ} \mathrm{C}$.

2.3. Determination of the Activity of Single Alkaloid. AntiMRSA activity of BAs was determined by broth microdilution

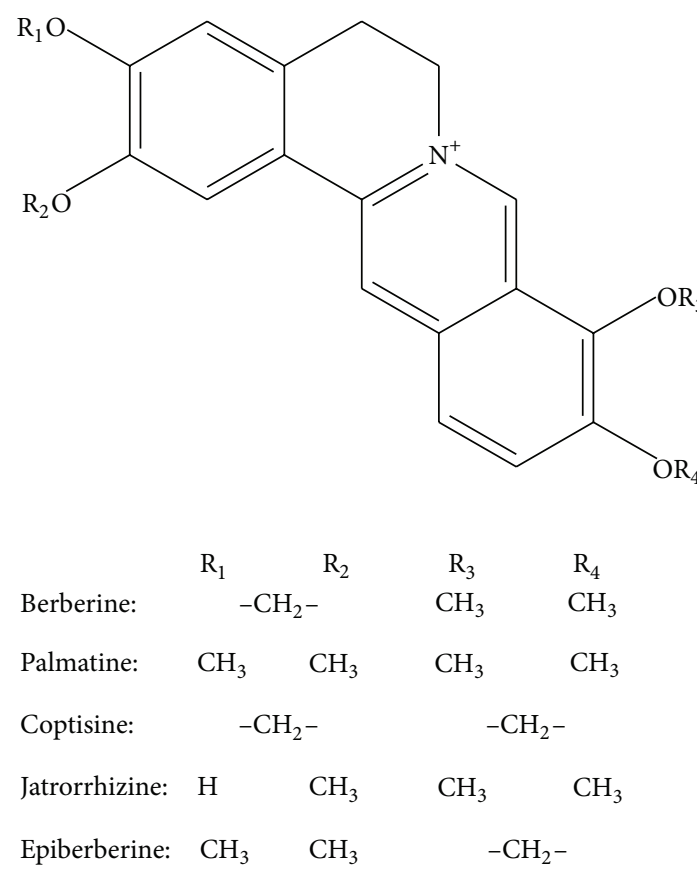

FIGURE 1: Molecular structures of berberine alkaloids.

according to a modified version of the Clinical and Laboratory Standards Institute (CLSI) protocol that included fluorescent determination using resazurin [19]. Resazurin is a blue dye that is nonfluorescent until it is reduced to the pink colored and highly red fluorescent resorufin. It has a large change in perceived color hue when the thickness or concentration of an observed sample increases or decreases. The fluorescence intensity of different growth stages of bacteria was determined by Synergy H1 hybrid multimode microplate reader (BioTek, Winooski, USA). The concentration of resazurin sodium salt (Sigma, Saint Louis, MO, USA) in the assay was $2.84 \mu \mathrm{g} / \mathrm{mL}$, and the excitation and emission wavelengths were set to $544 \mathrm{~nm}$ and $590 \mathrm{~nm}$, respectively. Fluorescence intensity was determined $17 \mathrm{~h}$ after the experiment (when the fluorescence intensity peaked). The concentrations of the extracts of BAs were 2048, 1024, $512,256,128,64,32,16,8$, and $4 \mu \mathrm{g} / \mathrm{mL}$ for rows $1-10$, respectively. Rows 11 and 12 were set as infected control (containing bacteria but no extract) and noninfected medium control (containing neither extract nor bacteria). Following fluorometric measurement, the $50 \%$ effective concentration $\left(\mathrm{EC}_{50}\right)$ was calculated using the fluorescence intensity of rows $1-10$ and the infected control.

2.4. Two-Component Combination Assay. The checkerboard assay was adopted for this assay. All 10 possible two-component combinations of these 5 active compounds were tested in duplicate in 36-point dose matrices (six doses for each compound, representing 720 data points) for their effects on the proliferation of MRSA. The five test concentrations for each compound were chosen by first determining the $\mathrm{EC}_{50}$ of each compound as a single agent in this assay, and then 
selecting 4-fold and 16-fold higher and lower concentrations, and the study is performed as Figure 2. Each combination was then scored to identify antibacterial effects that were greater than the effects of the individual components by using a resazurin proliferation assay. For each combination, we calculated the difference between the observed effect of each combination of doses and the predicted effect based on two models of additivism [20]. The highest single agent (HSA) model is the larger of the effects produced by each of the combination's single agents at the same concentrations as in the mixture. In contrast, the Bliss additivism model [21] predicts that the combined response $C$ for two single compounds with effects $\mathrm{A}$ and $\mathrm{B}$ is $\mathrm{C}=\mathrm{A}+\mathrm{B}-\mathrm{A} \times \mathrm{B}$, where each effect is expressed as fractional inhibition between 0 and 1. These effect-based synergy models make no assumptions about the functional form of the dose-response curves and do not require dose-response information that lies outside the range sampled by each screening matrix. To determine whether the readout of the combination therapy is additive, synergistic, or indifferent, we calculated the fractional inhibitory concentration indices (FICI) by the checkerboard assay. The FICI represents the sum of the FICs of each drug tested, where the FIC is determined for each drug by dividing the MIC of each drug when used in combination by the MIC of each drug when used alone:

$$
\begin{aligned}
\text { FICI }= & \left(\frac{\text { IIC }_{\text {drug A in combination }}}{\text { MIC }_{\text {drug A alone }}}\right) \\
& +\left(\frac{\mathrm{MIC}_{\mathrm{drug} \text { B in combination }}}{\mathrm{MIC}_{\mathrm{drug} \text { B alone }}}\right) .
\end{aligned}
$$

2.5. Multicomponent Combination Assay. The QRCD was adopted to investigate the multicomponent combination of BAs. In this study, the 5 components were set as 5 factors and each factor contained 5 levels (from 100 to $500 \mu \mathrm{g} / \mathrm{mL}$ with the interval of $100 \mu \mathrm{g} / \mathrm{mL}$ ) coded as $-2,-1,0,+1$, and +2 . The factor level table was designed for the experimental design of combination, and the inhibition ratios were calculated by determining the fluorescence of each combination test. The regression equation was established upon the principle of drug combination and the optimal combination could be obtained using Data Processing System (DPS, V12.01).

\subsection{Application and Validation of the Optimal Combina-} tion. The antimicrobial activity of the optimal combination obtained from this study was compared to three herbal medicines including Rhizoma Coptidis (the ratio of the alkaloids: Ber : Cop : Pal : Jat : Epi $=1: 0.23: 0.22: 0.09: 0.12)$, Phellodendri Chinensis (Ber: Pal: Jat $=1: 0.50: 0.06$ ), Berberidis Radix (Ber: Pal: Jat $=1: 0.70: 0.20$ ), and geometric proportion (Ber:Cop: Pal: Jat: Epi = 1:1:1:1:1). Improved broth microdilution was used to investigate the antimicrobial activity of different BA combinations on E. coli, S. aureus, S. dysenteriae, S. pneumonia, and C. albicans. The inhibition ratios were calculated to evaluate the antimicrobial activity of the combinations.

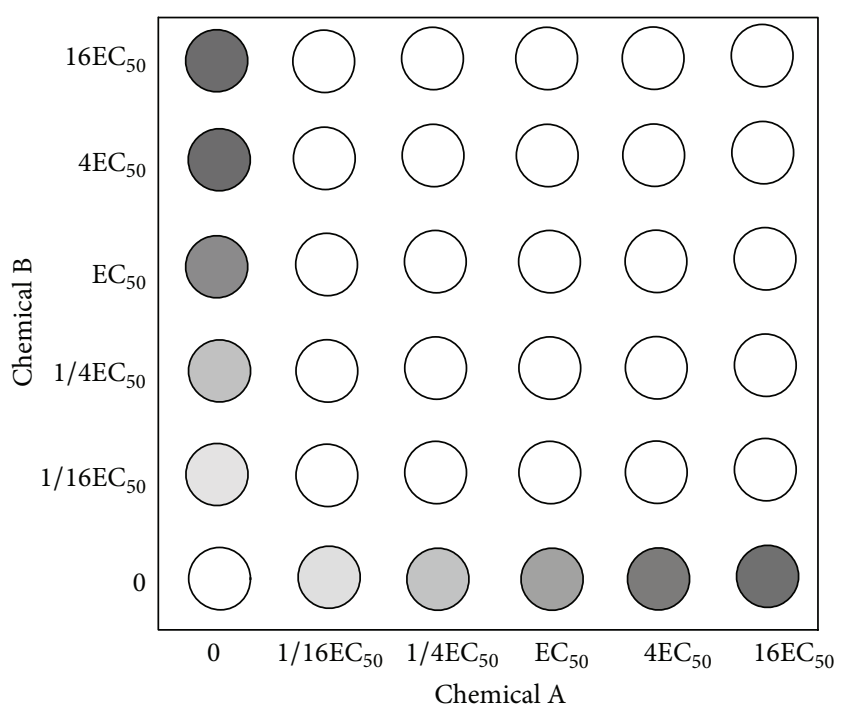

FIGURE 2: Schematic diagram of 36-point dose matrices for twocomponent combination assay. The five test concentrations for each compound were chosen by first determining the $\mathrm{EC}_{50}$ of each compound as a single agent in this assay, and then selecting 4-fold and 16 -fold higher and lower concentrations.

\subsection{Murine Model of Systemic Infection by Cyclophosphamide} $(C Y)$. Before infection, mice were rendered neutropenic by i.p. injection of BAs daily for 3 consecutive days at a dosage of $100 \mathrm{mg} / \mathrm{kg}$ body weight before inoculation. Mice were then infected with $0.1 \mathrm{~mL}$ of inoculum of MRSA $\left(2 \times 10^{6} \mathrm{cfu} / \mathrm{mL}\right)$ in warmed saline $\left(35^{\circ} \mathrm{C}\right)$ by the lateral tail vein on day 3 after pretreatment with BAs [22]. A group of mice were injected with $0.1 \mathrm{~mL}$ of $2 \times 10^{6} \mathrm{cfu} / \mathrm{mL}$ dead MRSA suspension per mouse as negative control. In addition, a group of mice were inoculated with $0.1 \mathrm{ml}$ of $2 \times 10^{6} \mathrm{cfu} / \mathrm{mL}$ suspensions per mouse without advance BAs treatment as negative controls. Confirmation of infection was determined in duplicate for final isolate candidates.

Data were averaged from three experiments. All groups of mice were observed 16 days after infection. At the end point, all surviving mice were killed by $\mathrm{CO}_{2}$ exposure. Different tissues of mice that died during the observation period and killed survivors were removed under aseptic conditions, which were subjected to detailed necropsy examinations. Kidneys, lungs, and brains of mice were homogenized in sterile $0.9 \%$ saline. Serial dilutions of the homogenates were plated onto sabouraud dextrose agar to calculate the cfus after $48 \mathrm{~h}$ of incubation at $35^{\circ} \mathrm{C}$.

2.8. Statistical Analyses. The data obtained from QRCD were analyzed using ANOVA, and the lack of fit test of regression equation was analyzed by using DPS (DPS, V12.01). The change in bacterial density in tissues, expressed as change in $\log _{10}$ cfu for both treated and untreated animals, was reported by using descriptive statistics. Effectiveness of the combinations was undertaken with appropriate statistical tests. The mean survival time (MST) data of each group 
of treated mice were compared with those from untreated controls by using one-way ANOVA. Student's $t$-test was used, and the significance level was defined as $P<0.01$.

\section{Results and Discussion}

3.1. Determination of the Activity of Single Alkaloid. The MRSA strain ATCC43300 contains mecA gene but no norA gene. In the absence of drug intervention, the fluorescence absorption reached around 40000 after 17 hours' incubation. In contrast, the fluorescence intensity was inhibited by adding the positive control cefoxitin and the alkaloids. The inhibiting effect could be calculated by the following equation: $I=$ $(A-X) / A \times 100 \%$, where $I$ represents the inhibiting ratio, $A$ represents the fluorescence absorption of the negative control (row 11), and $X$ represents the fluorescence absorptions of alkaloids groups (rows 1-10). After that, the $\mathrm{EC}_{50}$ values were calculated from the $I$-concentration curves. The result showed that, among the alkaloids, berberine showed the strongest antimicrobial activity (Figure 3), and the inhibiting ratios reached $85.2 \%$ and $94.0 \%$ at the concentration of $512 \mu \mathrm{g} / \mathrm{mL}$ and $1024 \mu \mathrm{g} / \mathrm{mL}$, respectively. The sequence of the activity of alkaloids was Ber $>$ Cop $>$ Jat $>$ Pal $>$ Epi.

This test adopted resazurin for fluorescent determination, and it could be found from Figure 4 that this method was reliable. When adding cefoxitin or Ber, the color of the MRSA suspension changed regularly with the increase of the concentration (Figures 4(a) and 4(b)); the fluorescence intensity at $24 \mathrm{~h}$ significantly decreased when adding $64 \mu \mathrm{g} / \mathrm{mL}$ cefoxitin (Figures 4(b) and 4(f)). In addition, it showed that the density of MRSA increased significantly after being incubated for $24 \mathrm{~h}$ (Figures 4(c) and 4(d)); however, the colony counts significantly decreased when adding $64 \mu \mathrm{g} / \mathrm{mL}$ cefoxitin or $128 \mu \mathrm{g} / \mathrm{mL}$ Ber (Figures $4(\mathrm{~g})$ and $4(\mathrm{~h})$ ).

To date, there have been some reports of drug combinations on MRSA, such as tannins and polyphenols [23], baicalein and ciprofloxacin [24], flavones and $\beta$-lactam antibiotics [25], usnic acid and antimicrobial agents [26], anacardic acids and methicillin [27], arbekacin and vancomycin [28], polyoxometalates and oxacillin [29], fosfomycin and arbekacin [30], gemcitabine and gentamicin [31], penicillin and Salix babylonica L. [32], ciprofloxacin and Isatis tinctoria L. [32], ceftriaxone and Scutellaria baicalensis G. [32], Rosmarinus officinalis and cefuroxime [33], and pannarin and antibiotics [34]. However, to our knowledge, there have not been any reports on the combinations of ingredients in traditional medicine. The present study showed that alkaloids had anti-MRSA activity, but the activity was relatively weak compared with antibiotics. The reason might be that the bacteria had multiple drug resistance which resulted in low absolute concentration inside the cell membrane. Bacteria have evolved numerous defenses against antimicrobial agents, and drug-resistant pathogens are on the rise [35]. A general and effective defense is conferred by ubiquitous multidrug resistance pumps (MDRs), membrane translocases that extrude structurally unrelated toxins from the cell [3639].

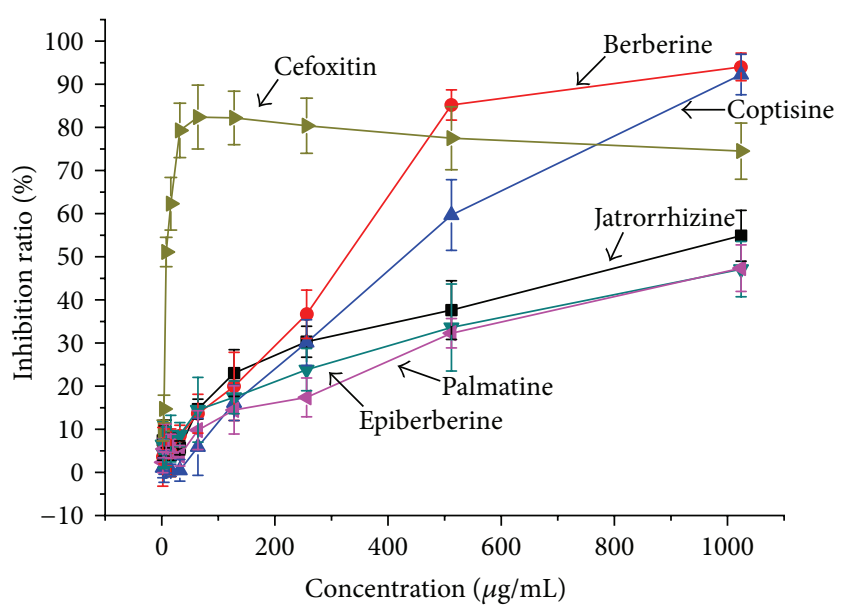

FIGURE 3: Inhibiting ratios of different concentrations of cefoxitin and five berberine alkaloids on MRSA.

3.2. Two-Component Combination Assay. Drug interactions generally consist of synergistic, additive, and antagonistic effects [40]. The interactions among BAs are marked by 6 color gradations, among which purple indicates strong antagonistic effect, orange indicates slight antagonistic effect, dark blue indicates additive effect, light blue indicates slight synergistic effect, brilliant yellow indicates strong slight synergistic effect, and green indicates strong synergistic effect. The results showed two regularities. Firstly, the interactions among BAs were related to their concentrations, and the interactions changed along with the change of the concentrations (Figures 5 and 6). For example, while the concentration of Ber was $310.5 \mu \mathrm{g} / \mathrm{mL}\left(\mathrm{EC}_{50}\right)$ and the concentration of Epi was $266.4 \mu \mathrm{g} / \mathrm{mL}\left(1 / 4 \mathrm{EC}_{50}\right), 1065.4 \mu \mathrm{g} / \mathrm{mL}\left(\mathrm{EC}_{50}\right)$, or $4261.6 \mu \mathrm{g} / \mathrm{mL}\left(4 \mathrm{EC}_{50}\right)$, the excess over HAS > 20\% and the excess over Bliss $>10 \%$; while the concentration of Ber was $19.4 \mu \mathrm{g} / \mathrm{mL}\left(1 / 16 \mathrm{EC}_{50}\right)$ and the concentration of Epi was $66.6 \mu \mathrm{g} / \mathrm{mL}\left(1 / 16 \mathrm{EC}_{50}\right)$, the excess over HAS $=-7 \%$ and the excess over Bliss $=-6 \%$. Secondly, different BAs showed different interactions; specifically, the combinations of Ber and Epi, Jat and Pal, Jat and Cop, Cop and Epi showed intensive synergistic effect within the test concentrations (MICs and FICIs were determined, and the result showed a synergistic effect on inhibiting CP with FICI $<0.5$ or $0.5<$ FICI $<1)$.

By combining HAS and Bliss additivism models, it can, to a large degree, avoid false-positive results. There are a number of algorithms for quantifying synergy in the screening experiments. For example, median effect and isobolographic analyses effectively identify combinations in which one drug enhances the potency of the other drug. From the 36-point dose matrix, it was illustrated that the interactions of BAs were relevant to the proportion of each component. For example, the combination Ber \& Epi showed strong synergistic effect while the proportion of Ber was $20 \%$; the combination Pal \& Epi showed obvious antagonistic effect while the proportion of Pal was $20 \%$. The interactions among the drugs are the foundation of exploiting multicomponent drugs, and the development of such drugs should retain synergistic 
TABLE 1: Quadratic rotation-orthogonal combination design and results.

\begin{tabular}{|c|c|c|c|c|c|c|}
\hline No. & JAT & COP & BER & EPI & PAL & Inhibiting ratio (\%) \\
\hline 1 & 1 & 1 & 1 & 1 & 1 & 90.6 \\
\hline 2 & 1 & 1 & 1 & -1 & -1 & 70.0 \\
\hline 3 & 1 & 1 & -1 & 1 & -1 & 50.7 \\
\hline 4 & 1 & 1 & -1 & -1 & 1 & 44.1 \\
\hline 5 & 1 & -1 & 1 & 1 & -1 & 66.3 \\
\hline 6 & 1 & -1 & 1 & -1 & 1 & 72.9 \\
\hline 7 & 1 & -1 & -1 & 1 & 1 & 30.0 \\
\hline 8 & 1 & -1 & -1 & -1 & -1 & 32.5 \\
\hline 9 & -1 & 1 & 1 & 1 & -1 & 43.0 \\
\hline 10 & -1 & 1 & 1 & -1 & 1 & 70.4 \\
\hline 11 & -1 & 1 & -1 & 1 & 1 & 52.3 \\
\hline 12 & -1 & 1 & -1 & -1 & -1 & 30.4 \\
\hline 13 & -1 & -1 & 1 & 1 & 1 & 61.9 \\
\hline 14 & -1 & -1 & 1 & -1 & -1 & 26.3 \\
\hline 15 & -1 & -1 & -1 & 1 & -1 & 37.3 \\
\hline 16 & -1 & -1 & -1 & -1 & 1 & 16.2 \\
\hline 17 & -2 & 0 & 0 & 0 & 0 & 20.9 \\
\hline 18 & 2 & 0 & 0 & 0 & 0 & 40.4 \\
\hline 19 & 0 & -2 & 0 & 0 & 0 & 29.3 \\
\hline 20 & 0 & 2 & 0 & 0 & 0 & 71.0 \\
\hline 21 & 0 & 0 & -2 & 0 & 0 & 23.9 \\
\hline 22 & 0 & 0 & 2 & 0 & 0 & 40.4 \\
\hline 23 & 0 & 0 & 0 & -2 & 0 & 17.8 \\
\hline 24 & 0 & 0 & 0 & 2 & 0 & 39.2 \\
\hline 25 & 0 & 0 & 0 & 0 & -2 & 9.5 \\
\hline 26 & 0 & 0 & 0 & 0 & 2 & 30.8 \\
\hline 27 & 0 & 0 & 0 & 0 & 0 & 23.8 \\
\hline 28 & 0 & 0 & 0 & 0 & 0 & 41.8 \\
\hline 29 & 0 & 0 & 0 & 0 & 0 & 42.8 \\
\hline 30 & 0 & 0 & 0 & 0 & 0 & 28.6 \\
\hline 31 & 0 & 0 & 0 & 0 & 0 & 39.5 \\
\hline 32 & 0 & 0 & 0 & 0 & 0 & 44.9 \\
\hline 33 & 0 & 0 & 0 & 0 & 0 & 39.0 \\
\hline 34 & 0 & 0 & 0 & 0 & 0 & 30.3 \\
\hline 35 & 0 & 0 & 0 & 0 & 0 & 41.5 \\
\hline 36 & 0 & 0 & 0 & 0 & 0 & 36.7 \\
\hline
\end{tabular}

proportions and exclude antagonistic proportions. Following this principle, the interactions of the 10 combinations were analyzed and the optimal proportion region was obtained: Ber : Cop : Pal : Jat : Epi $=4: 1-4: 4-16: 1-16: 1-4$.

3.3. Multicomponent Combination Assay. According to the factor level table, a total of 36 experiments were designed, and the protocol and results were shown in Table 1 . The regression equation was obtained by DPS: $Y=0.34807+0.06611 X_{1}+$ $0.07975 X_{2}+0.10040 X_{3}+0.04670 X_{4}+0.05185 X_{5}+$ $0.01532 X_{1}^{2}+0.06406 X_{2}^{2}+0.01898 X_{3}^{2}+0.00986 X_{4}^{2}-$ $0.01093 X_{5}^{2}-0.00044 X_{1} X_{2}+0.04814 X_{1} X_{3}-0.02066 X_{1} X_{4}-$ $0.02852 X_{1} X_{5}-0.00919 X_{2} X_{3}-0.01625 X_{2} X_{4}+0.02793 X_{2} X_{5}-$
TABLE 2: Frequency distribution of the 1685 protocols with inhibiting ratio larger than 0.41 .

\begin{tabular}{lcc}
\hline & Weighted average & $95 \%$ confidence intervals \\
\hline$X_{1}(\mathrm{JAT})$ & 0.363 & $0.297 \cdots 0.429$ \\
$X_{2}(\mathrm{COP})$ & 0.446 & $0.374 \cdots 0.519$ \\
$X_{3}(\mathrm{BER})$ & 0.517 & $0.455 \cdots 0.578$ \\
$X_{4}(\mathrm{EPI})$ & 0.254 & $0.187 \cdots 0.321$ \\
$X_{5}(\mathrm{PAL})$ & 0.272 & $0.207 \cdots 0.337$ \\
\hline
\end{tabular}

$0.01545 X_{3} X_{4}+0.06154 X_{3} X_{5}-0.00412 X_{4} X_{5}$. By the analysis of variance, it showed that the lack of fit test of regression equation $F 1=$ MS lack of fit $/ \mathrm{MS}$ error $=7.0009>F 0.01$ (6, $9), F 3=$ MS regression $/ \mathrm{MS}$ error $=9.33>F 0.01$, indicating significant. Therefore, the regression result was reliable.

The regression coefficient was tested for its significance, and the items that were not significant were eliminated at the level $\sigma=0.10$. The equation was optimized as $Y=$ $0.34807+0.06611 X_{1}+0.07975 X_{2}+0.10040 X_{3}+0.04670 X_{4}+$ $0.05185 X_{5}+0.06406 X_{2}^{2}$. Analyzed by DPS, the program numbers higher than 0.41 were 1685 . Meanwhile, the weighted mean and the 95\% confidence interval were calculated (Table 2). Standardized the weighted mean of berberine by 1 , the weighted means of other alkaloids were obtained, and the optimal ratio was obtained as follows: $X_{1}: X_{2}: X_{3}: X_{4}: X_{5}=$ $0.702: 0.863: 1: 0.491: 0.526$.

There have been multiple design methods, such as Central Composite Design [41], Box-Behnken Design [42], and Doptimal Design [43]. Compared with these methods, QRCD presents two features: (1) it sacrifices partial orthogonality to obtain rotatability, and it retains the merits of less test times, simple calculation, and low dependency among the regression coefficients; (2) it discards the disadvantage of lacking rotatability that cannot screen the optimal regions from predicted values. The results of this section were consistent to the results in 3.2 .

3.4. Application and Validation. Figure 7 showed that the optimal combination in 3.3 had the strongest inhibiting effects on E. coli, S. aureus, S. dysenteriae, and S. pneumonia. Specifically, the $\mathrm{EC}_{50}$ of geometric proportion of alkaloids on E. coli was 1.81 times larger than that of the optimal combination; the $\mathrm{EC}_{50}$ of Berberidis Radix on $S$. aureus was 1.74 times larger than that of the optimal combination; the $\mathrm{EC}_{50}$ of geometric proportion of alkaloids on $S$. dysenteriae was 1.59 times larger than that of the optimal combination; the $\mathrm{EC}_{50}$ of Berberidis Radix on S. pneumonia was 1.41 times larger than that of the optimal combination. In addition, the antimicrobial activity of the optimal combination on C. albicans ranked second only to Rhizoma Coptidis. The results indicated that the optimal combination was not only applicable for ATCC43300, but also other laboratory strains.

This study provided a fast and reliable way for the interaction research of chemicals, and it also laid a foundation for the development and application of multicomponent drugs. However, this study only involves in vitro tests, and the 


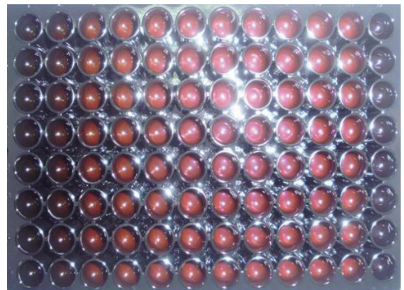

(a)

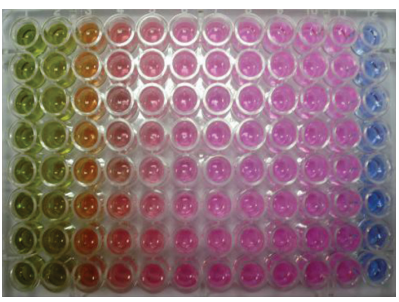

(e)

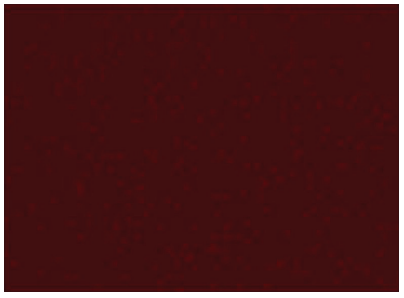

(b)

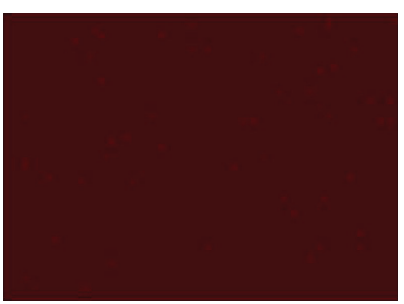

(f)

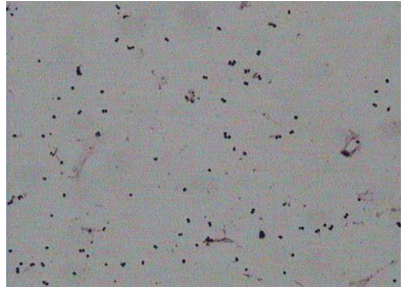

(c)

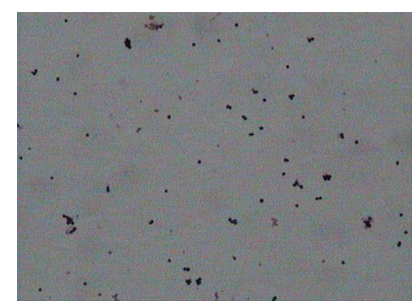

(g)

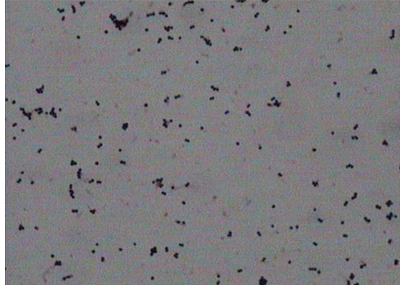

(d)

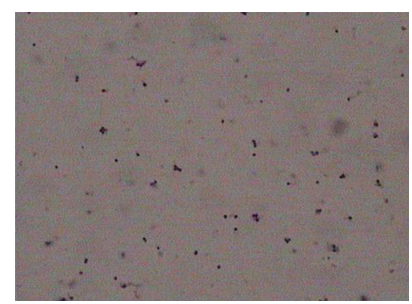

(h)

Figure 4: Fluorescent determination using resazurin. (a) 96-well fluorescent plates with 2-fold dilutions of Ber; (b) fluorescent image of MRSA suspension without any chemical; (c) the microphotograph of MRSA at $0 \mathrm{~h}$ without any chemical (stained with gram stain); (d) the microphotograph of MRSA at $24 \mathrm{~h}$ without any chemical; (e) 96-well translucent plates with 2-fold dilutions of Ber; (f) fluorescent image of MRSA suspension with $64 \mu \mathrm{g} / \mathrm{mL}$ cefoxitin; (g) the microphotograph of MRSA at $24 \mathrm{~h}$ with $64 \mu \mathrm{g} / \mathrm{mL}$ cefoxitin; (h) the microphotograph of MRSA at $24 \mathrm{~h}$ with $128 \mu \mathrm{g} / \mathrm{mL}$ Ber.

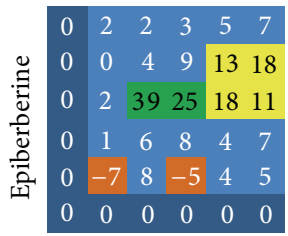

Berberine

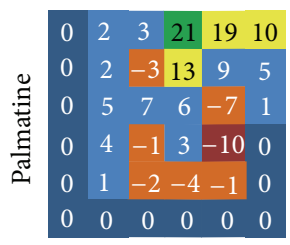

Epiberberine

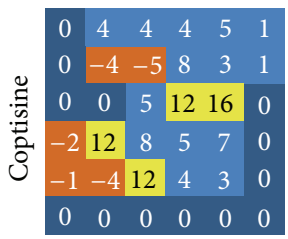

Berberine

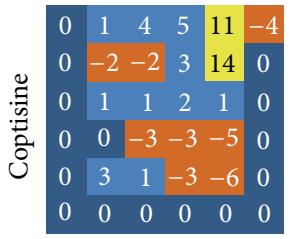

Epiberberine

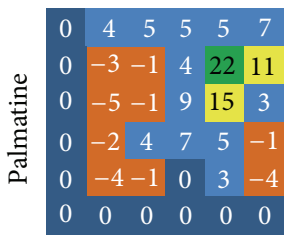

Berberine

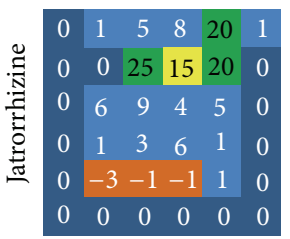

Coptisine

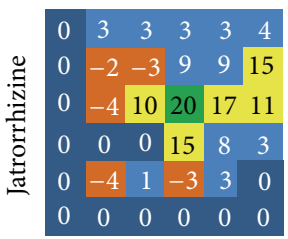

Berberine

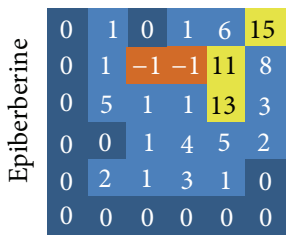

Jatrorrhizine

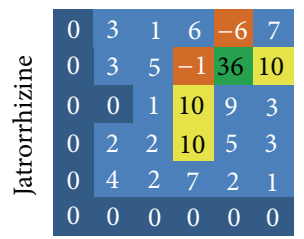

Palmatine

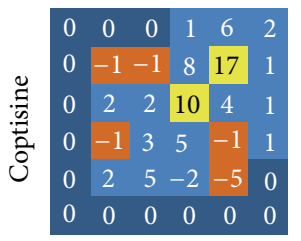

Palmatine

Figure 5: The excess over the highest single agent model, including 10 combinations of the 5 berberine alkaloids. Purple squares indicate strong antagonistic effect, orange squares indicate slight antagonistic effect, dark blue squares indicate additive effect, light blue squares indicate slight synergistic effect, brilliant yellow squares indicate strong slight synergistic effect, and green squares indicate strong synergistic effect.

exploitation of multicomponent drugs still needs the validation of in vivo experiments.

3.5. Establishment of an Antibacterial Mouse Model. The efficacy of the synergistic pairs was tested in a living immunocompromised animal model. We developed an immunocompromised mouse model by i.p. injection of cyclophosphamide at a dosage of $100 \mathrm{mg} / \mathrm{kg}$ (body weight) once daily for 3 consecutive days to specific pathogen-free female ICR mice. To test the model with pathogens, mice were infected with $0.1 \mathrm{~mL}$ of MRSA suspension of $2 \times 10^{5}$ blastospores per mouse in warmed saline $\left(35^{\circ} \mathrm{C}\right)$ by the lateral tail vein on day 3 after pretreatment with CY. We succeeded in causing $100 \%$ mortality within 6-8 days, which was suitable for subsequent drug-evaluation experiments. To test whether the BAs combinations had synergistic effects in the CYimmunocompromised mouse model, the test compound(s) either alone or in combination were administered orally by gavage $6 \mathrm{~h}$ postinfection and once daily thereafter for 5 days. A control group received $0.1 \mathrm{~mL}$ of saline by the same route as the placebo regimens. Organs of dead and killed mice at day 16 after infection were homogenized in sterile saline, diluted, 

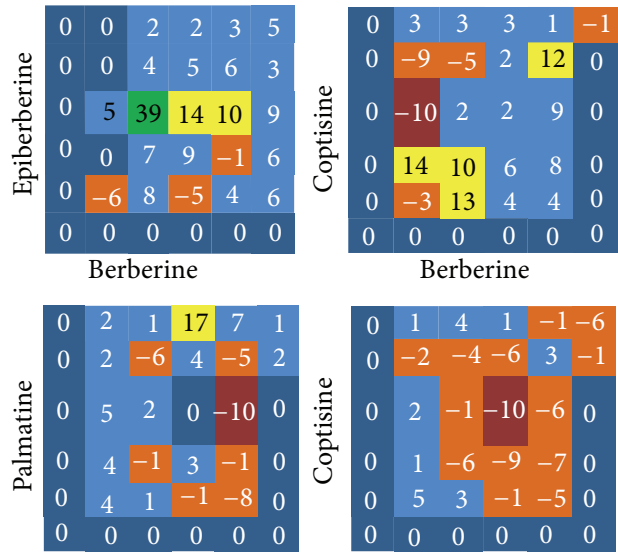

Epiberberine

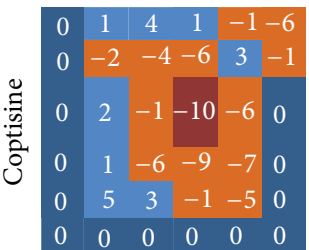

Epiberberine

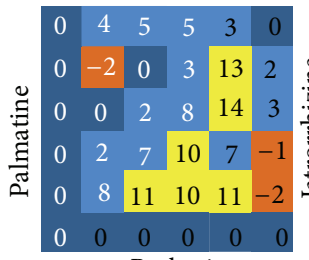

Berberine

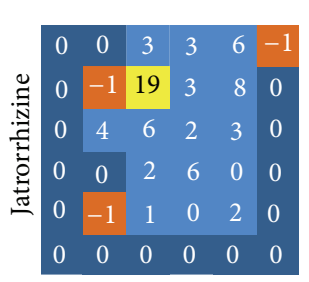

Coptisine

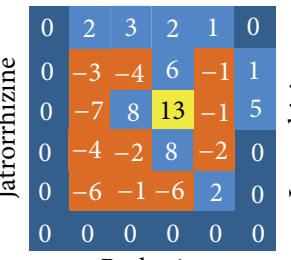

Berberine

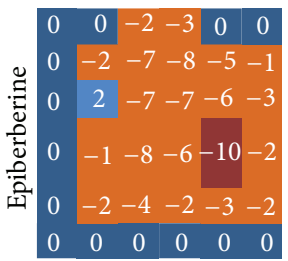

Jatrorrhizine

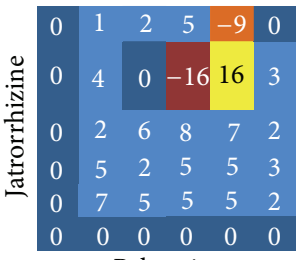

Palmatine

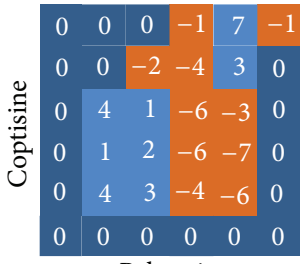

Palmatine

FIGURE 6: The excess over Bliss additivism model, including 10 combinations of the 5 berberine alkaloids.

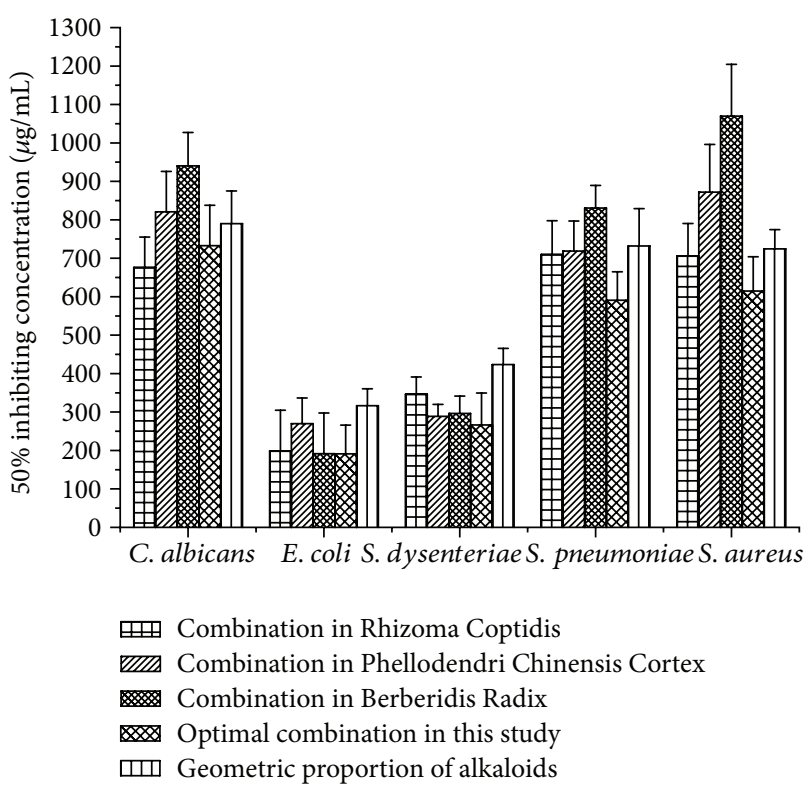

FIGURE 7: Comparison of the antimicrobial activities of different combinations of berberine alkaloids on five laboratory strains, including E. coli, S. aureus, S. dysenteriae, S. pneumonia, and C. albicans.

and spread onto sabouraud dextrose agar plates. Colony counts were determined after $48 \mathrm{~h}$ at $35^{\circ} \mathrm{C}$ for calculation of geometric means. Comparative efficacy assessed by changes in the bacterial density in kidney, lung, and brain tissue of the infected mice after treatment, percent survival, and MST were undertaken for test compound(s). Results obtained from Figure 8 showed that the combination in Phellodendri Chinensis Cortex and geometric proportion of alkaloids group did not have significant therapeutic effects on the body weight and percent survival of infected mice compared with placebo control $(P>0.05)$. The course of infection indicates that the MST was $5.1 \pm 0.5,6.8 \pm 0.6$, and $7.6 \pm 0.5$ for the placebo control, geometric proportion of alkaloids group, and combination in Phellodendri Chinensis Cortex group, respectively. On the contrary, the combination in Rhizoma Coptidis, cefoxitin, and optimal combination in this study group showed significant therapeutic effects $(P<0.01)$, in which the optimal combination in this study group showed the strongest anti-MRSA activity in vivo. The MST was $11.9 \pm$ 1.2 for the optimal combination group.

After being infected by MRSA, the body weight reduced compared with placebo control, and the hair became sparse (Figures 9(a) and 9(b)). By counting the bacterial colony, it were indicated that the remaining bacteria was determined in kidney, lung, and brain tissues. Among the combinations, cefoxitin, combination in Rhizoma Coptidis, and optimal combination in this study significantly reduced the $\left[\log _{10} \mathrm{cfu}\right.$ (cell/g)] compared with placebo control. For the optimal combination, the remaining bacteria $\left[\log _{10} \mathrm{cfu}(\mathrm{cell} / \mathrm{g})\right]$ in kidney, lungs, and brain reduced dramatically from 8.1 to 4.3 , 4.7 to 2.6 , and 2.8 to 1.8 , respectively (Figure $9(\mathrm{~g})$ ).

\section{Conclusions}

In summary, this study initially investigated the anti-MRSA activity of single berberine alkaloid on MRSA, and the individual $\mathrm{EC}_{50}$ was obtained. Thereafter, 4-fold and 16-fold higher and lower concentrations of $\mathrm{EC}_{50}$ were chosen for their interactions among berberine alkaloids by checkerboard assay, and the synergetic and antagonistic interactions were clarified. Next, the multi-component combination was studied by QRCD and the optimal combination for anti-MRSA activity was screened. In addition, the optimal combination was applied for its potency on other type strains, and it was demonstrated that the combination had a wide versatility. Furthermore, the BAs were tested on CYimmunocompromised mouse model to validate the therapeutic effects of the selected combinations. 


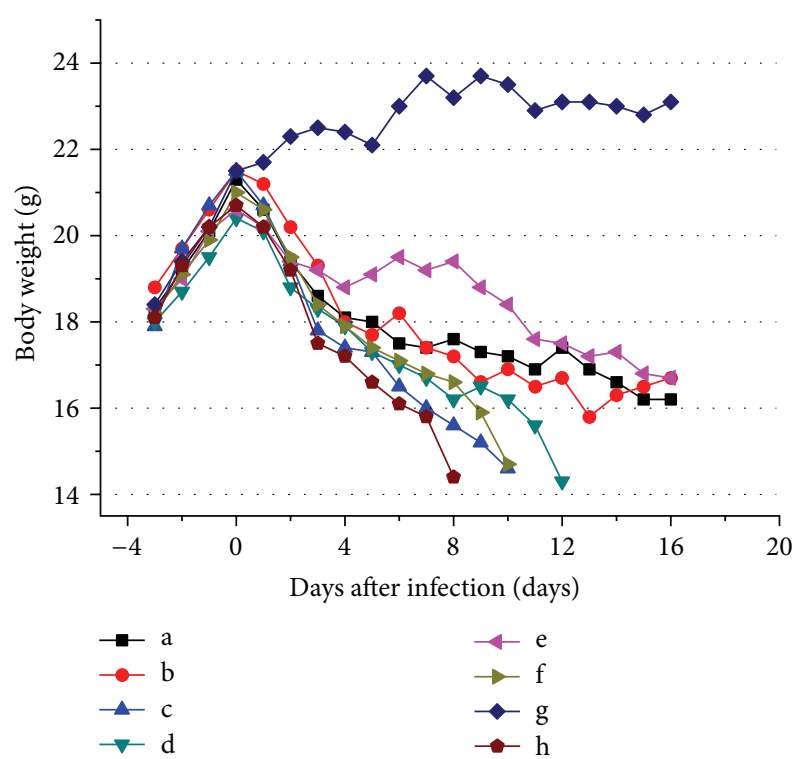

(a)

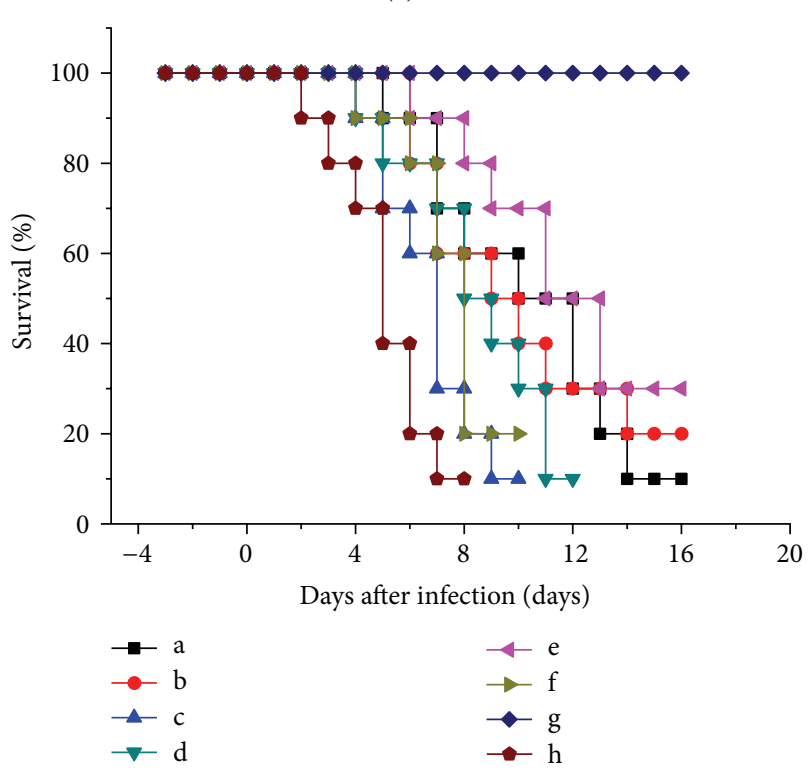

(b)

FIGURE 8: In vivo anti-MRSA effects of BAs combinations. (a) Body weight of ICR mice from day -3 to 16 ; (b) percent survival of ICR mice during the therapeutic process. (a: cefoxitin group; b: combination in Rhizoma Coptidis group; c: combination in Phellodendri Chinensis Cortex group; d: combination in Berberidis Radix group; e: optimal combination in this study group; $\mathrm{f}$ : geometric proportion of alkaloids group; g: negative group (noninfection mice without being given drugs); h: placebo control).

\section{Conflict of Interests}

The authors have declared that there is no conflict of interests.

\section{Authors' Contribution}

J. Luo and D. Yan contributed equally to this work.

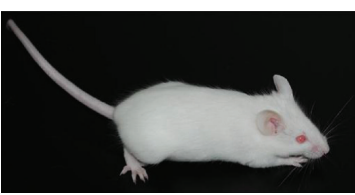

(a)

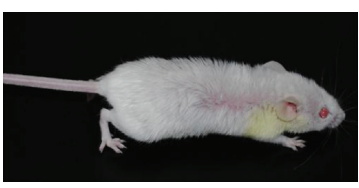

(b)

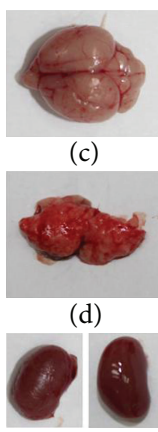

(e)
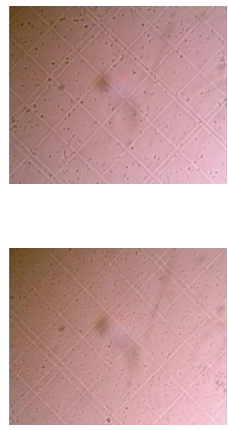

(f)

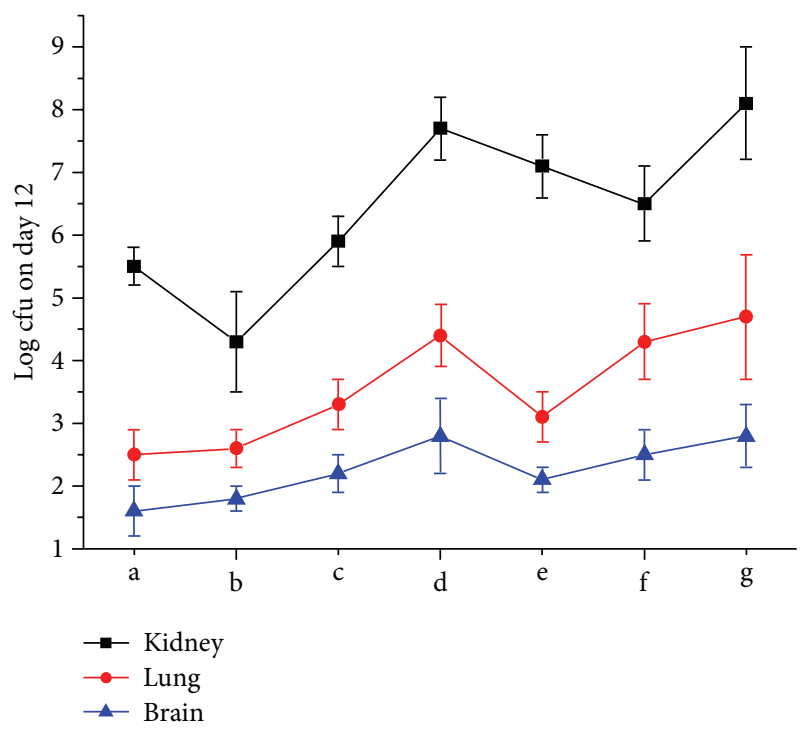

(g)

Figure 9: Bacterial density in kidney, lung, and brain tissues. (a) Pathogen-free female ICR mice; (b) MRSA-infected ICR mice; (c) brain tissue of died or killed mice; (d) lung tissue of died or killed mice; (e) kidney tissue of died or killed mice; (f) micrograph; (g) colony counts on day 12 (log cfu). (a: cefoxitin group; b: optimal combination in this study group; c: combination in Rhizoma Coptidis group; d: combination in Phellodendri Chinensis Cortex group; e: combination in Berberidis Radix group; f: geometric proportion of alkaloids group; g: placebo group).

\section{Acknowledgments}

The authors are grateful for the support from the National Science Foundation of China (81073043 and 81274078), the Major Subject of National Science and Technology (2009ZX09502-022), and the Open Research Fund of the State Key Laboratory Breeding Base of Systematic Research, Development and Utilization of Chinese Medicinal Resource.

\section{References}

[1] Y. Y. Yu, B. C. Wang, L. Peng, J. B. Wang, and C. Zeng, "Advances in pharmacological studies of Coptis chinesis," Journal of Chongqing University, vol. 29, no. 2, pp. 107-111, 2006.

[2] U. K. Choi, M. H. Kim, and N. H. Lee, "Optimization of antibacterial activity by gold-thread (Coptidis rhizoma Franch) against 
Streptococcus mutans using evolutionary operation-factorial design technique," Journal of Microbiology and Biotechnology, vol. 17, no. 11, pp. 1880-1884, 2007.

[3] H. Y. Kim, H. S. Shin, H. Park et al., "In vitro inhibition of coronavirus replications by the traditionally used medicinal herbal extracts, Cimicifuga rhizoma, Meliae cortex, Coptidis rhizoma, and Phellodendron cortex," Journal of Clinical Virology, vol. 41, no. 2, pp. 122-128, 2008.

[4] D. L. Fan, X. H. Xiao, and X. J. Ma, "Calorimetric study of the effect of protoberberine alkaloids in Coptis chinensis Franch on Staphylococcus aureus growth," Thermochimica Acta, vol. 480, no. 1-2, pp. 49-52, 2008.

[5] Y. S. Lee, W. S. Kim, K. H. Kim et al., "Berberine, a natural plant product, activates AMP-activated protein kinase with beneficial metabolic effects in diabetic and insulin-resistant states," Diabetes, vol. 55, no. 8, pp. 2256-2264, 2006.

[6] W. J. Kong, J. Wei, P. Abidi et al., "Berberine is a novel cholesterol-lowering drug working through a unique mechanism distinct from statins," Nature Medicine, vol. 10, no. 12, pp. 13441351, 2004.

[7] W. J. Kong, Y. L. Zhao, X. H. Xiao et al., "Spectrum-effect relationships between ultra performance liquid chromatography fingerprints and anti-bacterial activities of Rhizoma coptidis," Analytica Chimica Acta, vol. 634, no. 2, pp. 279-285, 2009.

[8] P. L. Ding, L. Q. Chen, Y. Lu, and Y. G. Li, "Determination of protoberberine alkaloids in Rhizoma coptidis by ERETIC ${ }^{1} \mathrm{H}$ NMR method," Journal Pharmaceutical and Biomedical Analysis, vol. 60, pp. 44-50, 2012.

[9] C. O. Chan, C. C. Chu, D. K. W. Mok, and F. T. Chau, "Analysis of berberine and total alkaloid content in Cortex Phellodendri by near infrared spectroscopy (NIRS) compared with highperformance liquid chromatography coupled with ultra-visible spectrometric detection," Analytica Chimica Acta, vol. 592, no. 2, pp. 121-131, 2007.

[10] G. Y. Zuo, G. C. Wang, Y. B. Zhao et al., "Screening of Chinese medicinal plants for inhibition against clinical isolates of methicillin-resistant Staphylococcus aureus (MRSA)," Journal of Ethnopharmacology, vol. 120, no. 2, pp. 287-290, 2008.

[11] R. Skov, K. Christiansen, S. J. Dancer et al., "Update on the prevention and control of community-acquired meticillin-resistant Staphylococcus aureus (CA-MRSA)," International Journal of Antimicrobial Agents, vol. 39, no. 3, pp. 193-200, 2012.

[12] E. Turos, G. S. K. Reddy, K. Greenhalgh et al., "Penicillin-bound polyacrylate nanoparticles: restoring the activity of $\beta$-lactam antibiotics against MRSA," Bioorganic and Medicinal Chemistry Letters, vol. 17, no. 12, pp. 3468-3472, 2007.

[13] D. Styers, D. J. Sheehan, P. Hogan, and D. F. Sahm, "Laboratorybased surveillance of current antimicrobial resistance patterns and trends among Staphylococcus aureus: 2005 status in the United States," Annals of Clinical Microbiology and Antimicrobials, vol. 5, article 2, 2006.

[14] S. R. Norrby, C. E. Nord, and R. Finch, "Lack of development of new antimicrobial drugs: a potential serious threat to public health," Lancet Infectious Diseases, vol. 5, no. 2, pp. 115-119, 2005.

[15] R. H. Deurenberg, C. Vink, S. Kalenic, A. W. Friedrich, C. A. Bruggeman, and E. E. Stobberingh, "The molecular evolution of methicillin-resistant Staphylococcus aureus," Clinical Microbiology and Infection, vol. 13, no. 3, pp. 222-235, 2007.

[16] C. R. Bonapace, J. A. Bosso, L. V. Friedrich, and R. L. White, "Comparison of methods of interpretation of checkerboard synergy testing," Diagnostic Microbiology and Infectious Disease, vol. 44, no. 4, pp. 363-366, 2002.
[17] H. P. Wei, X. L. Ye, H. Y. Zhang, X. G. Li, and Y. J. Zhong, "Investigations on cordycepin production by solid culture of Cordyceps militaris," Zhongguo Zhong Yao Za Zhi, vol. 33, no. 19, pp. 2159-2162, 2008 (Chinese).

[18] P. Zheng, L. J. Meng, G. P. Sun, and W. Z. Wang, "Study on optimization of formulation of Danggui Liuhuang effervescent granules," Zhong Yao Cai, vol. 34, no. 3, pp. 452-455, 2011 (Chinese).

[19] Clinical and Laboratory Standards Institute, Methods for Dilution Antimicrobial Susceptibility Tests for Bacteria that Grow Aerobically, Approved Standard, vol. 32, Clinical and Laboratory Standards Institute, Wayne, Pa, USA, 9th edition, 2012.

[20] A. A. Borisy, P. J. Elliott, N. W. Hurst et al., "Systematic discovery of multicomponent therapeutics," Proceedings of the National Academy of Sciences of the United States of America, vol. 100, no. 13, pp. 7977-7982, 2003.

[21] M. C. Berenbaum, "Criteria for analyzing interactions between biologically active agents," Advances in Cancer Research, vol. 35, pp. 269-335, 1981.

[22] L. X. Zhang, K. Z. Yan, Y. Zhang et al., "High-throughput synergy screening identifies microbial metabolites as combination agents for the treatment of fungal infections," Proceedings of the National Academy of Sciences of the United States of America, vol. 104, no. 11, pp. 4606-4611, 2007.

[23] S. Mandal, M. DebMandal, N. K. Pal, and K. Saha, "Synergistic anti-Staphylococcus aureus activity of amoxicillin in combination with Emblica officinalis and Nymphae odorata extracts," Asian Pacific Journal of Tropical Medicine, vol. 3, no. 9, pp. 711714, 2010.

[24] B. C. Chan, M. Ip, C. B. Lau et al., "Synergistic effects of baicalein with ciprofloxacin against NorA over-expressed methicillinresistant Staphylococcus aureus (MRSA) and inhibition of MRSA pyruvate kinase," Journal of Ethnopharmacolgy, vol. 137, no. 1, pp. 767-773, 2011.

[25] Y. Sato, H. Shibata, T. Arai et al., "Variation in synergistic activity by flavone and its related compounds on the increased susceptibility of various strains of methicillin-resistant Staphylococcus aureus to beta-lactam antibiotics," International Journal of Antimicrobial Agents, vol. 24, no. 3, pp. 226-233, 2004.

[26] B. Segatore, P. Bellio, D. Setacci et al., "In vitro interaction of usnic acid in combination with antimicrobial agents against methicillin-resistant Staphylococcus aureus clinical isolates determined by FICI and $\Delta \mathrm{E}$ model methods," Phytomedicine, vol. 19, no. 3-4, pp. 341-347, 2012.

[27] H. Muroi, K. I. Nihei, K. Tsujimoto, and I. Kubo, "Synergistic effects of anacardic acids and methicillin against methicillin resistant Staphylococcus aureus," Bioorganic and Medicinal Chemistry, vol. 12, no. 3, pp. 583-587, 2004.

[28] I. You, R. Kariyama, M. J. Zervos, H. Kumon, and J. W. Chow, "In-vitro activity of arbekacin alone and in combination with vancomycin against gentamicin- and methicillin-resistant Staphylococcus aureus," Diagnostic Microbiology and Infectious Disease, vol. 36, no. 1, pp. 37-41, 2000.

[29] M. Inoue, T. Suzuki, Y. Fujita et al., "Synergistic effect of polyoxometalates in combination with oxacillin against methicillinresistant and vancomycin-resistant Staphylococcus aureus: a high initial inoculum of $1 \times 10^{8} \mathrm{cfu} / \mathrm{ml}$ for in vivo test," Biomedicine and Pharmacotherapy, vol. 60, no. 5, pp. 220-226, 2006.

[30] K. Morikawa, M. Nonaka, Y. Yoshikawa, and I. Torii, "Synergistic effect of fosfomycin and arbekacin on a methicillinresistant Staphylococcus aureus-induced biofilm in a rat model," 
International Journal of Antimicrobial Agents, vol. 25, no. 1, pp. 44-50, 2005.

[31] L. P. Jordheim, S. Ben Larbi, O. Fendrich et al., "Gemcitabine is active against clinical multiresistant Staphylococcus aureus strains and is synergistic with gentamicin," International Journal of Antimicrobial Agents, vol. 39, no. 5, pp. 444-447, 2012.

[32] Z. C. Yang, B. C. Wang, X. S. Yang, Q. Wang, and L. Ran, "The synergistic activity of antibiotics combined with eight traditional Chinese medicines against two different strains of Staphylococcus aureus," Colloids and Surfaces B, vol. 41, no. 2-3, pp. 79-81, 2005.

[33] N. Jarrar, A. Abu-Hijleh, and K. Adwan, "Antibacterial activity of Rosmarinus officinalis L. alone and in combination with cefuroxime against methicillin-resistant Staphylococcus aureus," Asian Pacific Journal of Tropical Medicine, vol. 3, no. 2, pp. 121123, 2010.

[34] G. Celenza, B. Segatore, D. Setacci et al., "In vitro antimicrobial activity of pannarin alone and in combination with antibiotics against methicillin-resistant Staphylococcus aureus clinical isolates," Phytomedicine, vol. 19, no. 7, pp. 596-602, 2012.

[35] S. B. Levy, "The challenge of antibiotic resistance," Scientific American, vol. 278, pp. 46-53, 1998.

[36] K. Lewis, "Multidrug resistance efflux," in Transport of Molecules Across Microbial Membranes, J. K. Broome-Smith, S. Baumberg, C. J. Stirling, and F. B. Ward, Eds., pp. 15-40, Cambridge University Press, Cambridge, UK, 1999.

[37] H. Nikaido, "Multiple antibiotic resistance and efflux," Current Opinion in Microbiology, vol. 1, no. 5, pp. 516-523, 1998.

[38] I. T. Paulsen, M. H. Brown, and R. A. Skurray, "Proton-dependent multidrug efflux systems," Microbiological Reviews, vol. 60, no. 4, pp. 575-608, 1996.

[39] J. S. Lolkema, B. Poolman, and W. N. Konings, "Bacterial solute uptake and efflux systems," Current Opinion in Microbiology, vol. 1, no. 2, pp. 248-253, 1998.

[40] E. Kuley and F. Özogul, "Synergistic and antagonistic effect of lactic acid bacteria on tyramine production by food-borne pathogenic bacteria in tyrosine decarboxylase broth," Food Chemistry, vol. 127, no. 3, pp. 1163-1168, 2011.

[41] V. V. Guaracho, N. M. S. Kaminari, M. J. J. S. Ponte, and H. A. Ponte, "Central Composite experimental design applied to removal of lead and nickel from sand," Journal of Hazardous Materials, vol. 172, no. 2-3, pp. 1087-1092, 2009.

[42] S. L. Ferreira, R. E. Bruns, H. S. Ferreira et al., "Box-Behnken design: an alternative for the optimization of analytical methods," Analytica Chimica Acta, vol. 597, no. 2, pp. 179-186, 2007.

[43] R. Morales, M. C. Ortiz, L. A. Sarabia, and M. S. Sánchez, "Doptimal designs and $\mathrm{N}$-way techniques to determine sulfathiazole in milk by molecular fluorescence spectroscopy," Analytica Chimica Acta, vol. 707, no. 1-2, pp. 38-46, 2011. 


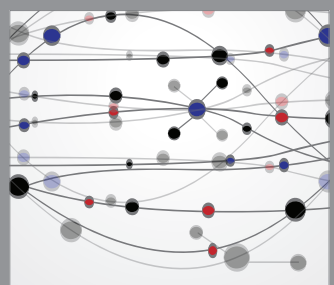

The Scientific World Journal
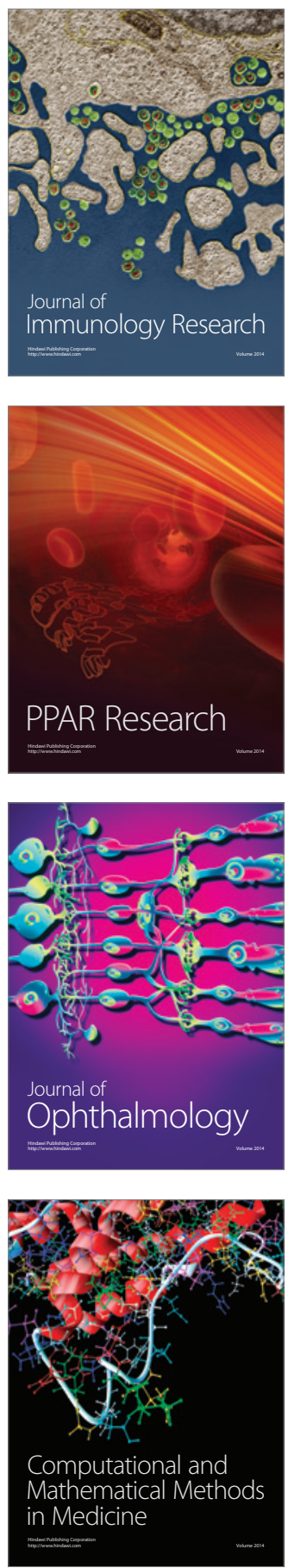

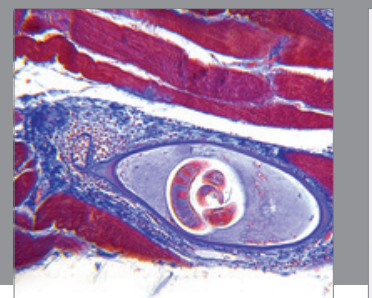

Gastroenterology

Research and Practice
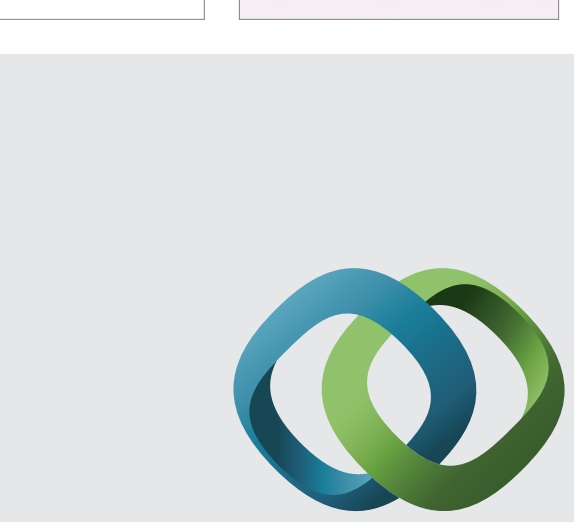

\section{Hindawi}

Submit your manuscripts at

http://www.hindawi.com
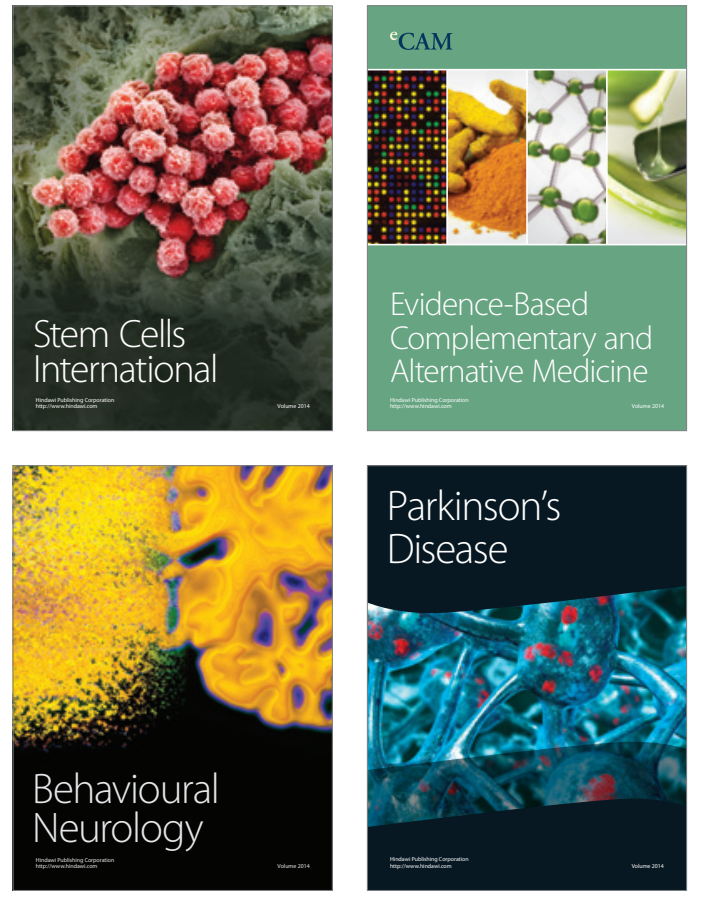
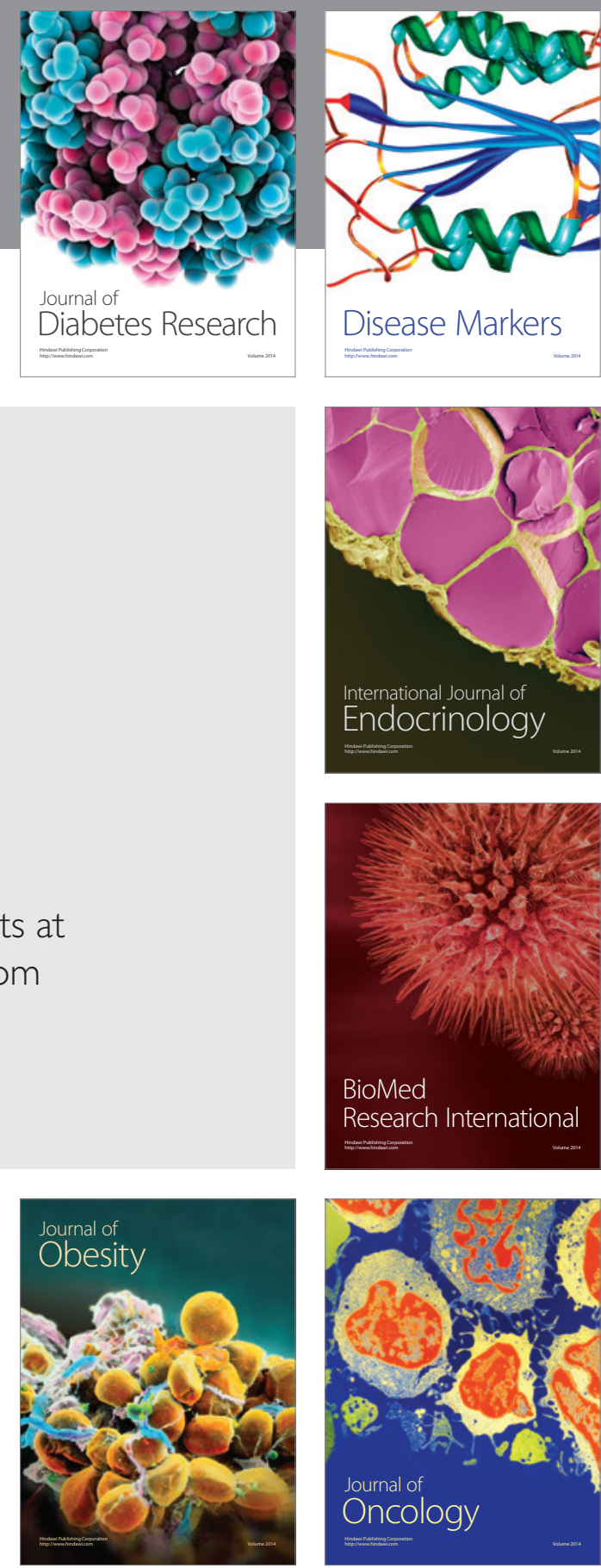

Disease Markers
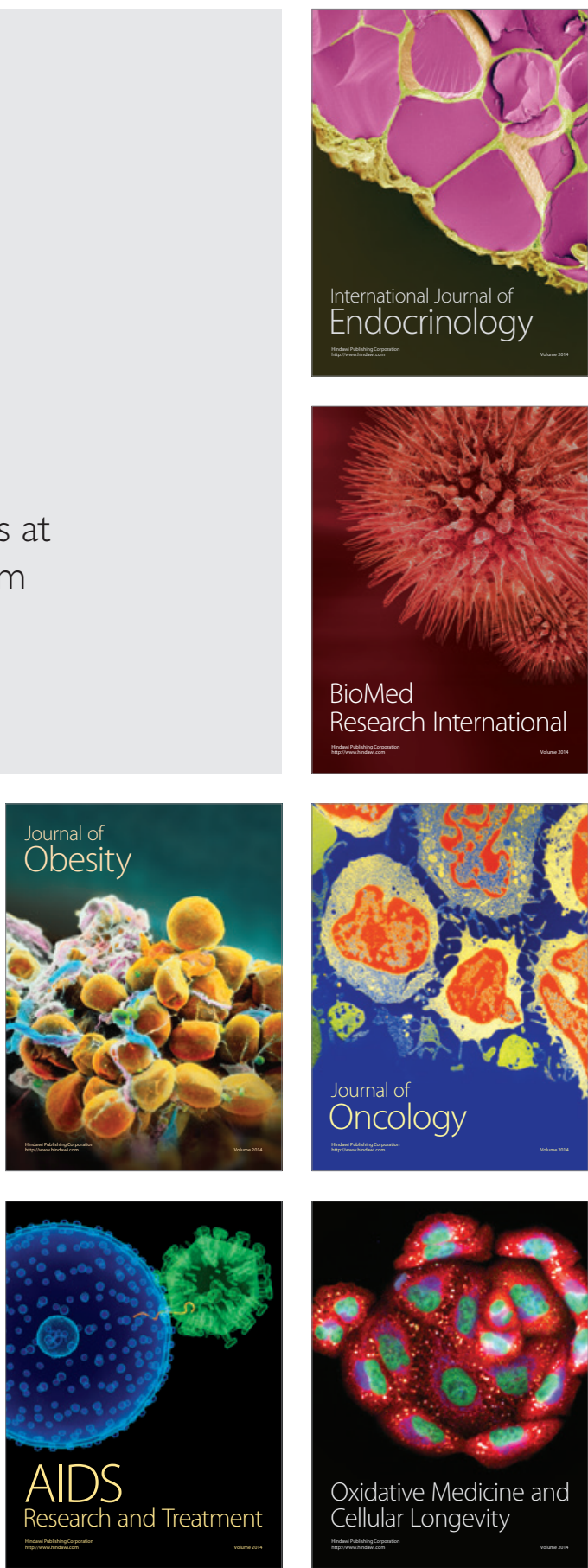